\title{
Development of a grooming process for an agile software team in the automotive domain
}

\begin{abstract}
At the current unpredictable technical evolution, the market is demanding an increasingly flexibility from companies to adapt to the pace of change in what customers want. The present research was developed in an automotive company, where software teams are pursuing Agile methodologies to coupe with these challenges. Teams use the Scrum framework, however, lack of efficient communication among team members results in poor performance of the product owner and the development team. In an attempt to solve this issue and according to the needs shared by the teams, this paper proposes a grooming process for a Scrum team. It provides a step-wise approach to work breakdown, from customer requirements elicitation to the development of ready work entities using the user story format. This paper describes how agile methods can support requirements engineering in a software project.
\end{abstract}

Keywords: Agile Methodology; Scrum; Product Backlog Grooming.

\section{Introduction}

One of the main concerns of business leaders is how to deal with the present unstable business environment, known as VUCA - Volatile, Uncertain, Complex and Ambiguous world. Coined by the US Army College on the 90s, this acronym "reflects an increasingly unstable and rapidly changing business world" [1]. To fulfil this need, the Agile methodology was created in 2001 by a group named Agile Manifesto. This methodology deals with continuous value delivery, incrementally, welcoming changing requirements, communications, and sustainability, among others [2]. Its main principle is being adaptable and iterative, following the Inspect and Adapt cycle [3].

Scrum is the most used Agile framework in the world for organizing and managing work, combining specific roles, artefacts, and ceremonies. The Product Owner, more related to the project manager role, is the most difficult role due to the responsibility for maintaining and prioritizing the work [4]. 
The company where this research took place is part of the automotive domain. Due to its dimension and complexity, software teams at the development hub face a huge problem: inefficient communication. To date, the responsibility of the work breakdown and long-term planning belonged to the Product Owner, not considering inputs from the development team. Most of the times, teams receive low level engineering tasks, not allowing the team's involvement in the requirements breakdown process from customer requirements to small work packages ready to be implemented. The teams share, even more, the necessity and willingness to participate in the work breakdown, giving their contribution on the best way to achieve a milestone and giving inputs for the work prioritization. If the teams were able to accomplish this, they would be improving their autonomy and commitment, which would make them more mature and efficient as a team, bringing also more value to the project.

However, this breakdown and prioritization of work - named the grooming process in Scrum - is not a mathematical process and there are many approaches in literature. This process breaks out customer requirements, acquired from the stakeholders, into specific work for the team to perform in one work cycle. Considering the need and desire shared by the teams, the main goal of this paper is to define a grooming process, that will initiate with the collection of customer requirements until ready to develop entities are available to the teams. Given the size and complexity of the organization and the large number of teams at the hub dealing with the stated problem, it was necessary to reduce the sample size to only a single team of 5 developers on which this research acted upon. This decision was motivated by the availability of a specific team to adopt new work methods and approaches. To reach the goal, it was crucial to have a deep involvement of the participating team, once they are the ones that need to implement new practices in order to improve their performance. Taking this into account, the Action Research methodology was selected to better fit on the organization needs [5]. Briefly, it constitutes a cycle of diagnosis, action, and evaluation, in which the main intent is to drive research projects with strong involvement of the researcher into the companies.

This paper is structured in five sections. In Literature Review section, the main knowledge areas involved are presented. Methodology section introduces the methodology used and the adjustments needed to guide this research work. The Results section starts with the motivation obtained from the diagnose phase of the action research cycle, and then describes the iterations performed where the last one is described in more detail. In each iteration the action research phases are explicitly referenced. Finally, conclusions are drawn concerning the learnings acquired during the development of the grooming process and also its implementation. Also, suggestions for future work are presented. 


\section{Literature Review}

\subsection{Scrum overview}

Scrum is one of the most popular Agile frameworks being used all over the world by Information Technologies (IT) companies. It concerns to a management and control process derived from "knowledge management, complex adaptive systems and empirical process control theory" [2], which aims to address complexity and developing software that satisfies business needs. It is used to organize and manage work, combining specifics roles, artefacts and ceremonies.

The Scrum team includes a Scrum Master (SM), a Product Owner (PO) and the Development Team (Dev. Team), presented in Fig. 1. The SM represents the team, being their coach or facilitator, assuring the team follows Scrum to achieve their highest performance level [6]. The PO on the other hand represents the business, users, and customers, working at guiding the team to the right goal. Finally, the Dev. Team is responsible for the product's development, considering the PO requests.

The heart of Scrum is each one of the small iterations of work, called Sprints, which are time-boxed generally with the same duration, and with the aim of delivering a product increment. Thus a new sprint starts immediately after the end of the previous one. Fig. 1 provides an overview of the Scrum work cycle. It starts with a meeting with the stakeholders, where the Project Vision is settled. At that point, the Product Backlog (PB) is created and prioritized by the PO. This PB is basically a prioritized list of requested project functionalities, also named Product Backlog Items (PBIs), represented on the left side of Fig. 1. These PBIs have different sizes and can be product features, User Stories (US) or EPICS (big user stories). A US can be described as a product increment unit ready to be estimated and developed in a sprint and includes in its description the Definition of Done (DoD), being similar to a checklist that guarantees the user story is really finished [7].

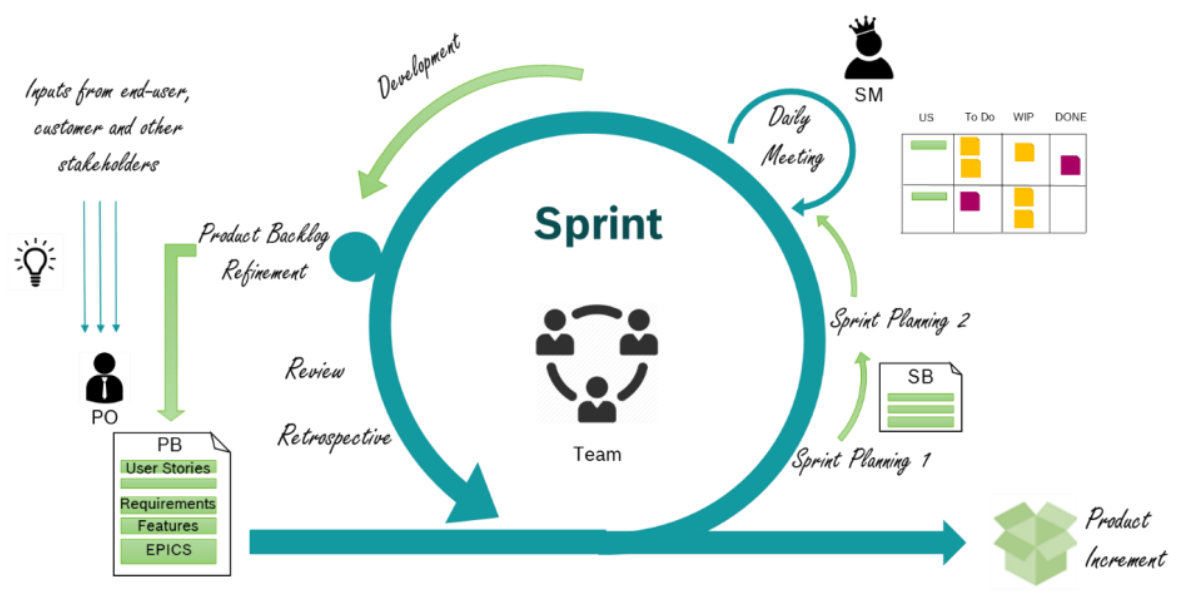

Fig. 1. Scrum cycle (Author's own elaboration) 
The sprint cycle begins with the Sprint Planning, divided into two different phases, where the team decides which work will be included in the sprint, once the PB could represent weeks or even months of work. The first half of planning concerns the requirements and answers to what the team should deliver at the end of the sprint. The second half of the meeting concerns to a high-level design, and answers to how the team will do the work $[2,8]$.

During the sprints, a Daily Meeting occurs with 15-minute maximum duration, to synchronize the team. At this moment, the team members share what they have done, what they plan to do next, obstacles they are facing and highlights, to keep the motivation high.

In each sprint, the Scrum team works to create an increment or a shippable product, entitled Accepted Deliverables. Before the end of the sprints, stakeholders and Scrum team meet in the Sprint Review meeting to check the increment delivered to assure the PO approves and accepts it.

The Sprint Retrospective meeting is the last ceremony, which concerns more to the processes the team follows to perform their work and relationships, in the same way the Sprint Review concerns the product. It is an opportunity for the team to inspect themselves, reflecting and sharing what went well and what did not [9].

\subsection{Product Backlog Grooming}

The PB grooming is not a ceremony considered on the Scrum cycle but many authors as Rubin [10] and Sutherland [8] consider it essential and valuable for a good, updated and organized PB. The PB grooming is nothing more than a meeting between the PO and the Scrum team (Dev. team and SM) to discuss the PB. This time-box meeting is an opportunity for the PO to share user stories and new features with the team. It aims to contribute and discuss future work in order to manage, organize and keep the PB updated. It is a collaborative effort, which focuses on three main activities, according to Rubin [10]:

- Creating and refining PB items - which includes activities to add, remove, update or split PBIs;

- Estimating PBIs;

- $\quad$ Prioritizing PBIs.

Considering a high rate of requirements changes, it is really important that these meetings with the team occur continuously, so the PB could follow and reflect these changes. At this point, refining the $\mathrm{PB}$ can include not only changes in the user stories' scope but also in their priority [11].

The capacity required is not a rule and differs between authors. Some of them defend the teams should invest at least $10 \%$ of each sprint capacity in grooming, like Rubin [10], and others defend that it should happen every week, as Leffingwell [12].

The main goal is to obtain US's ready to enter in a sprint, facilitating also the sprint planning, once it allows the team to have a better perception of what is supposed to be done and its value. Therefore, it contributes to transparency, team commitment (since they are involved), and decreasing of miscommunication and missed assumptions. For the PO it is an essential ceremony to have an overview of what is coming, and to build more reliable time plans, being the PB like a pipeline of requirements [10]. 


\section{Methodology}

The methodology adopted was Action Research, which assumes the collaboration and involvement of the researcher in the organization in which he is acting. This approach is based on five phases of the methodology described by Susman and Evered [5] and later defined by O'Brien [13]: Diagnosing, Action Planning, Taking Action, Evaluating and Specify Learnings.

The first phase - Diagnosing - is where observation and analysis take place and in which the Action Planning will be built upon. In the latter phase, the development of new tools, processes, templates and guidelines is expected, in order to increase the teams' efficiency. All these planned ideas will be implemented in a defined time frame, which concerns to the next phase: Taking Action. The fourth is the Evaluating phase, which assess the consequences of the implemented actions, with the objective of comparing the current state with the initial state, evidencing whether or not the proposed objectives were initially achieved. Finally, in Specify Learnings phase, main lessons learned that were assimilated are presented, more specifically the general findings related to the topic diagnosed and its improvements.

For the development of the grooming process, an initial version of the process was drafted and later implemented. This first version was subject of improvements along the development sprints performed by the team, supervised by the researcher. This continuous analysis (similar to the cycle inspect and adapt from the Agile Methodology) was performed within a single action research iteration. Thus, when following the action research cycle, during the Evaluation phase, problems with in the proposed grooming process were identified. At this point, it did not seem reasonable to develop either the learnings or a diagnose iteration due to the level of new findings acquired. The decision came to "short circuit" the action research cycle by performing several iterations of Taking Action, Evaluating and Action Planning within one action research iteration. A single instance of Diagnosing and Specify Learnings steps was performed, as described in Fig. 2.

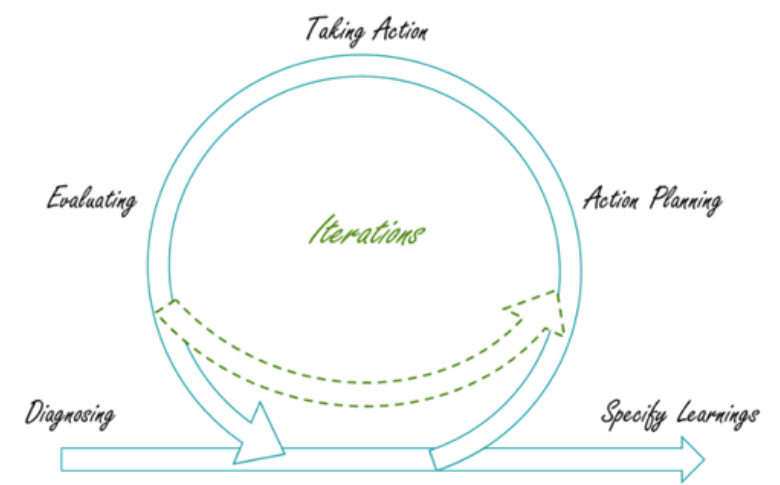

Fig. 2. Action research adapted cycle (author's own elaboration) 


\section{Results}

The team chosen as subject of research to develop the process had not adopted any grooming ceremony into their sprint cycle before. It was necessary to initiate this ceremony firstly and then, as stated before, to iterate over it until achieving a stable approach to the team's grooming process. To guide the effort the researcher joined the team assuming the role of scrum master.

The team used, as engineering guiding model, the V-model to define the expected outcomes for each user story available in the backlog. This means that each US available to the team had explicit outcomes of what was expected to be done in order to fulfil each of the V-Model asses, including: Mock-up creation, Product Requirements Elicitation, Design/Architecture definition, Implementation, Test. Taking these existing practices into account, the ones that related to the scope of the grooming process were mock-up creation and requirements elicitation.

\subsection{Iteration 1}

(Planning) For the first iteration, it was considered only one grooming meeting per sprint where only one team member was involved, avoiding spending too much resource capacity, and also to minimize the impact on team performance. This member was responsible for the mock-up development, considering an EPIC made available by the PO.

(Taking Action) At this point, the main goal of the grooming meeting was to validate the mock-up that serves as the basis for future implementation and to create a document with the respective product requirements. Often, doubts were expected to be clarified with the PO; in cases where he was not able to answer, he collected the questions and documented all the uncertainties to clarify later with the stakeholders.

(Evaluating) As result of this first iteration the need to make explicit the necessity to plan capacity for grooming arose, and secondly, the need of including another team member into the grooming process in order to allow all team members to participate in the process was reached.

\subsection{Iteration 2}

(Planning) To fulfil the need identified during iteration 1, the PO would now be responsible for creating a time box for sprint grooming. This approach reserved capacity of the team to ensure the grooming process was performed. As well, the time box included a description of the expected result from the customer requirement analysis, allowing the validation with the mock-up developed. As stated as essential, it was decided to involve another team member in a rotation scheme, ensuring that all team members participate directly in the process, enabling the sharing and guidance through the learning process. It also forces all members to work with the engineering practices related to grooming, such as the development of mock-ups and requirements development, allowing them to be in touch with different abstraction levels of software engineering. 
(Taking Action) The changes were implement and the grooming meeting was performed by two team members. However, at the end of the grooming meeting the expected outcome was not completely finished. At this point, it did not seem very helpful to end the sprint without ending the expected result from the grooming. To address this situation, a new grooming meeting was scheduled, allowing the team to share the modifications performed based on the outputs of the first meeting. So, during this interval between the meetings, the team was expected to update the mockup and associated requirements.

(Evaluating) The planed modifications, reserve capacity and adding a second team member were perceived as positive. Additionally to the planned change, an issue was identified related the effort needed to deliver the expected outcome. To address this issue an additional change to the process was tested. At the end of this iteration, a new problem was identified. The expectation was the requirements development relative to the customer requirements under analysis would be closed within the grooming performed in the sprint. The conclusion was that product requirements development needed additional effort to the effort initially planned.

\subsection{Iteration 3}

(Planning) For the third iteration two changes were planned. The first change was to add an explicit activity to improve the mockup, demanding also a second meeting to validate the outcome. A second change was to keep the product requirements development in the process, but at this time the expectation would be not to finish their development. This activity would be performed in a dedicated requirements workshop out of the scope of the grooming process.

(Taking Action) The changes were implemented as planned. At the end of this iteration, several product features were indented as result of the mockup development; the decision was to extend the grooming process to user story creation and not to continuously iterate between customer requirements and mockups.

(Evaluating) In this sense, the team decided to invest more time on building user stories for the customer requirement under analysis, even if it exceeded the sprint timeframe, then to start a new customer requirement breakdown. At this point of the process development, it was visible the necessity to change the initial scope of the grooming process. The last iteration already addressed other activities outside the grooming meeting initial defined for the grooming process. This required to expand the process definition to address the main goal of this research.

\subsection{Iteration 4}

(Planning) At this point of process development, and considering that the last iteration did not describe the complete process, it was needed to broaden the scope process to include all activities performed from customer requirements to user story creation. The work that was performed out of the meeting was not fully visible. This did not allow the team and the stakeholders to have the macro vision of the process, once it did not translate the real work performed specifically. 
(Taking Action) Together with the Product Owner a brainstorm was performed where it was possible to define a complete view of the process with all the knowledge collected at this point. The final result is illustrated in the activities diagram depicted bellow in Fig. 3.

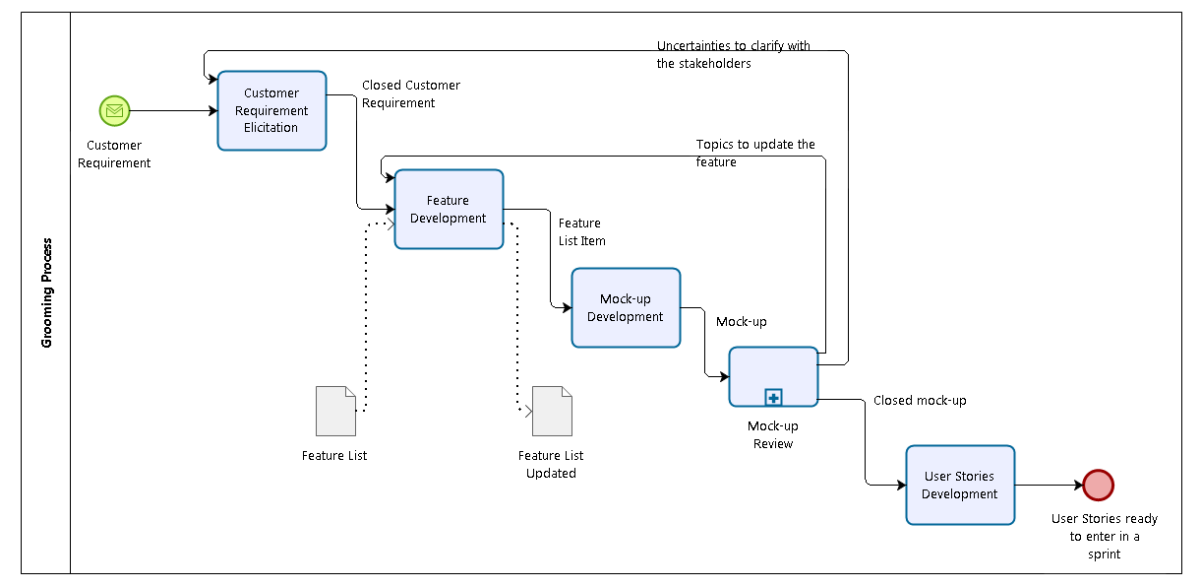

Fig. 3. Activities diagram of the grooming process.

The Customer Requirement (CR) is the trigger to start the process, and it is usually sent in a textual format. The first activity is the Customer Requirement Elicitation, where the team and mainly the Product Owner discuss with the stakeholders, allowing the comprehension of their needs. This is a decisive step, where the expectations from both parts are aligned and set. The output of this activity are agreed and closed CRs allowing to enter in the Feature Development activity. Here, the CR is mature enough to be translated to product features to be included in the product feature list. Usually the responsible for this activity is the Product Owner, but the team can also participate. One of the product features, a feature list item, is the input the team uses to develop a mock-up. This mock-up is the input for the Mock-up Review subprocess, more precisely the grooming meeting earlier established, where the output is the mock-up reviewed, when agreed. However, and as mentioned before, it can come back as input to previous activities, if unable to fulfill expectations. Only when it achieves the goal of the product features, the mock-up proceeds forward for the User Stories Development activity. Here, the user stories will be created and matured, which is the main output of the grooming process, in order to be ready to enter a sprint. The mock-up review sub-process, illustrated in Fig. 4, is the grooming meeting described previously, but now adapted to be part of the whole grooming process. Its exit points match exactly with the exit outputs of the sub-process Mock-up Review of Fig. 3. Similarly, the initial point of this sub-process is the same as the input illustrated in Fig. 3. The sub-process is represented in Fig. 4. 


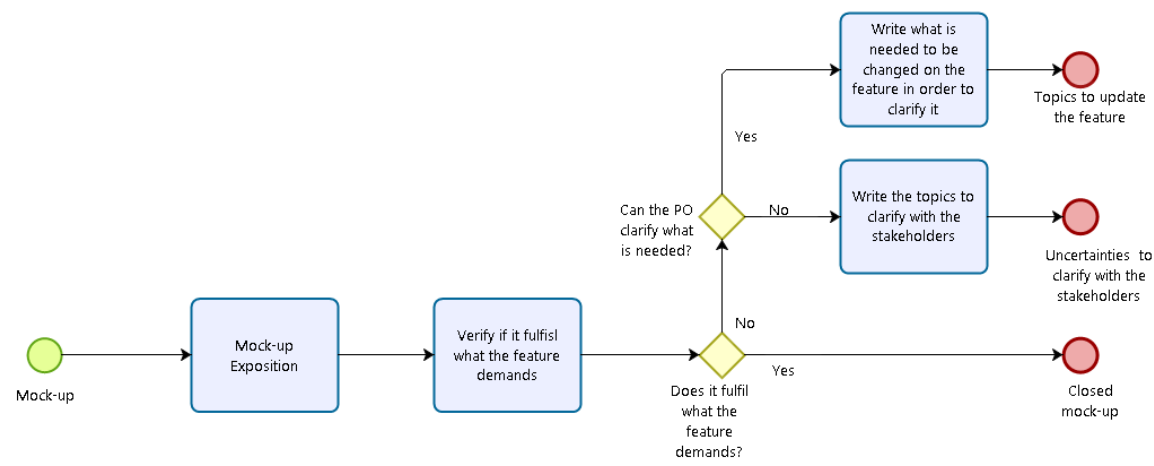

Fig. 4. Mock-up review meeting workflow

On the mock-up review meeting, three exit points exist. The desired exit is to end the meeting with a mock-up reviewed and settled to progress in the process. However, most of the times there are updates to be included in the mockups. As Fig. 4 shows, it is possible to have the Product Owner's feedback and just update the feature and the mock-up, by returning to the Feature Development activity, in order to define more accurate product features. In the worst case, when this meeting is not sufficient, it is also possible to return to the Requirements Elicitation activity to discuss with the stakeholders what is needed. Therefore, there is no limit how often the mock-up should be iterated. The purpose is to have a reliable mock-up that fulfils the customer requirements.

\section{Conclusions and Future Work}

(Evaluating and Specify Learnings)

During the execution of the action research cycle several findings were collected that resulted in improvements to the grooming process in each iteration. Despite the findings have not been so explicit and emphasized, at the end some conclusions and learnings could be considered and highlighted.

One of the main learnings was that there should be no binding between the grooming process and the sprint iteration. During the grooming process evolution, the process started with only one meeting for each sprint, with the purpose of creating the mockup, reviewing it and developing the US on the same sprint. However the conclusion was that this was not viable in the current setting of the team. The "when" and "how often" the meetings should happen is not the most important determining factor in setting up the grooming. Instead, it is important to assure they occur as many times as needed to guarantee the creation of User stories aligned with customer requirements. The balance of PBIs and team velocity determines the frequency of execution of the grooming process. This is possible because the grooming process is not bound to the development sprint but instead driven by the number of ready to implement user stories in the product backlog. This constituted an improvement to the PO and the team, since the process allowed a systematic approach to feed the product backlog. 
With a good backlog size, the PO can have a clear vision of the product roadmap and therefore improve ability to plan for a longer period than it was possible before.

Overall, the grooming process had a positive impact in how the team performed the engineering process. The grooming supported a systematic approach by the team in translating customer requirements to be ready to implement work units for each development sprint. This improved the maturity of the user stories created, ensuring a better alignment with customer requirements and also better sized to fit a development sprint. This resulted in improved ability to plan the sprint, decreasing the planning time and also the estimation accuracy between the planned and real work performed. Future work includes deployment of the grooming approach to other software development teams of the department.

\section{Acknowledgements}

This work is supported by: European Structural and Investment Funds in the FEDER component, through the Operational Competitiveness and Internationalization Programme (COMPETE 2020) [Project $\mathrm{n}^{\circ}$ 002797; Funding Reference: POCI-010247-FEDER-002797]

\section{References}

1. Lawrence, K.: Developing Leaders in a Business. UNC Exec. Dev. 1-15 (2013).

2. Hundermark, P.: Do-Better-Scrum. agile 42 - The Agile Coaching Company (2015).

3. Dean, B.J., Pessanha, B.G., Langfeldt, N., Pritchard, S., Stanger, J.: Agile Project Management with Scrum. (2006).

4. Sverrisdottir, H.S., Ingason, H.T., Jonasson, H.I.: The Role of the Product Owner in Scrum-comparison between Theory and Practices. Procedia - Soc. Behav. Sci. 119, 257-267 (2014).

5. Susman, G.I., Evered, R.D.: An assessment of the scientific merits of action research. Adm. Sci. Q. 23, 582-603 (1978).

6. Cohn, M.: Scum.

7. Mahnič, V., Hovelja, T.: On using planning poker for estimating user stories. J. Syst. Softw. 85, 2086-2095 (2012).

8. $\quad$ Sutherland, J.: Scrum handbook. Scrum Trining Intitute Press (2010).

9. Tobergte, D.R., Curtis, S.: A Guide to the Scrum Body of Knowledge. (2013).

10. Rubin, K.S.: Essential Scrum. Pearson Education, Inc. (2012).

11. Crisp, C., McKenna, D.: The Art of Scrum: How Scrum Masters Bind Dev Teams and Unleash Agility. (2016).

12. Leffingwell, D.: Agile Software Requirements: Lean Requirements Practices for Teams, Programs, and the Enterprise. (2011).

13. O'Brien, R.: An Overview of the Methodological Approach of Action Research. Univ. Toronto. (1998). 